\title{
(Non)Commutative Hopf Algebras of Trees and (Quasi)Symmetric Functions
}

\author{
Michael E. Hoffman \\ Dept. of Mathematics \\ U. S. Naval Academy, Annapolis, MD 21402 USA \\ meh@usna.edu
}

November 2, 2018

Keywords: Connes-Kreimer Hopf algebra, rooted trees, planar rooted trees, quasi-symmetric functions, Dyson-Schwinger equations MR Classfications: Primary 05C05, 05E05, 16W30; Secondary 81T15

\begin{abstract}
The Connes-Kreimer Hopf algebra of rooted trees, its dual, and the Foissy Hopf algebra of planar rooted trees are related to each other and to the well-known Hopf algebras of symmetric and quasi-symmetric functions via a pair of commutative diagrams. We show how this point of view can simplify computations in the Connes-Kreimer Hopf algebra and its dual, particularly for combinatorial Dyson-Schwinger equations.
\end{abstract}

\section{Introduction}

Hopf algebra techniques were introduced into the study of renormalization in quantum field theory by Connes and Kreimer [6]. The Hopf algebra defined by Connes and Kreimer (in its undecorated form), denoted here by $\mathcal{H}_{K}$, is the free commutative algebra on the rooted trees, with a coproduct that can be described in terms of "cuts" of rooted trees (see $\S 4$ below). The Hopf algebra $\mathcal{H}_{K}$ is the graded dual of another Hopf algebra (which we call $k \mathcal{T}$ ), studied earlier by Grossman and Larson [13], whose elements are rooted trees with a noncommutative multiplication. 
A noncommutative version of $\mathcal{H}_{K}$, denoted here by $\mathcal{H}_{F}$, was introduced by Foissy [7]: unlike $\mathcal{H}_{K}$, it is self-dual. As shown by Holtkamp [15], $\mathcal{H}_{F}$ is isomorphic to the Hopf algebra $k\left[Y_{\infty}\right]$ of planar binary trees defined by Loday and Ronco [16]. Foissy [8] showed $\mathcal{H}_{F}$ isomorphic to the "photon" Hopf algebra $\mathcal{H}^{\gamma}$ defined by Brouder and Frabetti [3, 4] in connection with renormalization. Here we define a Hopf algebra $k \mathcal{P}$, based on planar rooted trees in the same way $k \mathcal{T}$ is based on rooted trees, which is isomorphic to $\mathcal{H}_{F}^{*} \cong \mathcal{H}_{F}$. Our main purpose is to show how calculations in $\mathcal{H}_{K}$ and $k \mathcal{T}$ can be simplified by "lifting" them to $\mathcal{H}_{F} \cong k \mathcal{P}$.

After establishing a result on duality of graded connected Hopf algebras in $\S 2$, we briefly introduce in $\S 3$ some Hopf algebras familiar in combinatorics: the Hopf algebras Sym of symmetric functions, QSym of quasi-symmetric functions [11, and NSym of noncommutative symmetric functions [10]. Then we discuss, in parallel fashion, the Hopf algebras $k \mathcal{T}$ and $\mathcal{H}_{K}$ in $\S 4$, and $k \mathcal{P}$ and $\mathcal{H}_{F}$ in $\S 5$. In $\S 6$ we relate all these Hopf algebras by a pair of commutative diagrams, which we then apply to some calculations. First (in §6.1) we discuss families of elements of $k \mathcal{T}$ that parallel some familiar symmetric functions, and show how symmetric-function identities can be used to obtain results for rooted trees. Then in $\S 6.2$ we exhibit explicit solutions of some combinatorial Dyson-Schwinger equations in $\mathcal{H}_{K}$, and show that these solutions generate sub-Hopf-algebras of $\mathcal{H}_{K}$. Similar results on Dyson-Schwinger equations were obtained by Bergbauer and Kreimer [1] using different methods.

\section{Graded Connected Hopf Algebras}

Let $\mathcal{A}$ be a unital algebra (associative but not necessarily commutative) over a field $k$ of characteristic 0 . We assume $\mathcal{A}$ is graded, i.e.,

$$
\mathcal{A}=\bigoplus_{n \geq 0} \mathcal{A}_{n}
$$

with $\mathcal{A}_{n} \mathcal{A}_{m} \subset \mathcal{A}_{n+m}$. Necessarily $1 \in \mathcal{A}_{0}$ : we shall assume that $\mathcal{A}$ is connected, that is, $\mathcal{A}_{0}=k 1$.

A coalgebra structure on $\mathcal{A}$ consists of linear functions $\epsilon: \mathcal{A} \rightarrow k$ (counit) and $\Delta: \mathcal{A} \rightarrow \mathcal{A} \otimes \mathcal{A}$ (coproduct), such that $\epsilon$ sends $1 \in \mathcal{A}_{0}$ to $1 \in k$ and

all positive-degree elements of $\mathcal{A}$ to 0 , and $\Delta$ respects the grading. These functions must satisfy

$$
\left(\operatorname{id}_{\mathcal{A}} \otimes \epsilon\right) \Delta=\left(\epsilon \otimes \operatorname{id}_{\mathcal{A}}\right) \Delta=\operatorname{id}_{\mathcal{A}} .
$$


We also assume that $\Delta$ is coassociative, in the sense that $\Delta\left(\Delta \otimes \mathrm{id}_{\mathcal{A}}\right)=$ $\Delta\left(\operatorname{id}_{\mathcal{A}} \otimes \Delta\right)$. For $\mathcal{A}$ to be a Hopf algebra, $\Delta$ must be a homomorphism of graded algebras.

Writing the comultiplication applied to $u \in \mathcal{A}$ as

$$
\Delta(u)=\sum_{u} u^{\prime} \otimes u^{\prime \prime}
$$

we note that condition (2.1) requires that it have the form

$$
u \otimes 1+\sum_{\left|u^{\prime}\right|,\left|u^{\prime \prime}\right|>0} u^{\prime} \otimes u^{\prime \prime}+1 \otimes u .
$$

If $\Delta(u)=u \otimes 1+1 \otimes u$, then $u$ is primitive.

A Hopf algebra $\mathcal{A}$ has an antipode $S: \mathcal{A} \rightarrow \mathcal{A}$, which is an antiautomorphism of $\mathcal{A}$ with the properties that $S(1)=1$ and

$$
\sum_{u} S\left(u^{\prime}\right) u^{\prime \prime}=\sum_{u} u^{\prime} S\left(u^{\prime \prime}\right)=0
$$

for any $u$ of positive degree, where $u^{\prime}, u^{\prime \prime}$ are given by (2.2). Hence $S(u)=-u$ if $u$ is primitive. If $\mathcal{A}$ is either commutative or cocommutative (i.e., $T \Delta=\Delta$, where $T(a \otimes b)=b \otimes a)$, then $S^{2}=\mathrm{id}_{\mathcal{A}}$.

All the Hopf algebras we consider are locally finite, i.e., $\mathcal{A}_{k}$ is finitedimensional for all $k$. It follows that the (graded) dual $\mathcal{A}^{*}$ of $\mathcal{A}$ is also a Hopf algebra, with multiplication $\Delta^{*}$ and coproduct $\mu^{*}$ (where $\mu: \mathcal{A} \otimes A \rightarrow \mathcal{A}$ is the product on $\mathcal{A}$ ). The Hopf algebra $\mathcal{A}$ is cocommutative if and only if $\mathcal{A}^{*}$ is commutative.

By an inner product on a graded connected Hopf algebra $\mathcal{A}$, we mean a nondegenerate symmetric linear function $(\cdot, \cdot): \mathcal{A} \otimes \mathcal{A} \rightarrow k$ such that $(a, b)=0$ for homogeneous $a, b \in \mathcal{A}$ of different degrees. The following result gives a criterion to establish when two Hopf algebras $\mathcal{A}$ and $\mathcal{B}$ are dual (i.e., $\left.\mathcal{A}^{*} \cong \mathcal{B}\right)$.

Theorem 2.1. Let $\mathcal{A}, \mathcal{B}$ be graded connected locally finite Hopf algebras over $k$ which admit inner products $(\cdot, \cdot)_{\mathcal{A}}$ and $(\cdot, \cdot)_{\mathcal{B}}$ respectively. Then $\mathcal{A}$ and $\mathcal{B}$ are dual Hopf algebras provided there is a degree-preserving linear map $\phi: \mathcal{A} \rightarrow \mathcal{B}$ such that, for all $a_{1}, a_{2}, a_{3} \in \mathcal{A}$,

(a) $\left(a_{1}, a_{2}\right)_{\mathcal{A}}=\left(\phi\left(a_{1}\right), \phi\left(a_{2}\right)\right)_{\mathcal{B}}$; 
(b) $\left(a_{1} a_{2}, a_{3}\right)_{\mathcal{A}}=\left(\phi\left(a_{1}\right) \otimes \phi\left(a_{2}\right), \Delta\left(\phi\left(a_{3}\right)\right)\right)_{\mathcal{B}}$;

(c) $\left(a_{1} \otimes a_{2}, \Delta\left(a_{3}\right)\right)_{\mathcal{A}}=\left(\phi\left(a_{1}\right) \phi\left(a_{2}\right), \phi\left(a_{3}\right)\right)_{\mathcal{B}}$.

Proof. Define a linear function $\chi: \mathcal{B} \rightarrow \mathcal{A}^{*}$ by $\langle\chi(b), a\rangle=(b, \phi(a))_{\mathcal{B}}$. Injectivity follows from nondegeneracy of the inner products, and since $\mathcal{A}$ and $\mathcal{B}$ are locally finite it follows that $\chi$ is a bijection. It remains to show $\chi$ a homomorphism, i.e.,

$$
\left\langle\chi(\Delta(b)), a_{1} \otimes a_{2}\right\rangle=\left\langle\chi(b), a_{1} a_{2}\right\rangle \quad \text { for all } b \in \mathcal{B} \text { and } a_{1}, a_{2} \in \mathcal{A}
$$

and

$$
\left\langle\chi\left(b_{1} b_{2}\right), a\right\rangle=\left\langle\chi\left(b_{1}\right) \otimes \chi\left(b_{2}\right), \Delta(a)\right\rangle \quad \text { for all } b_{1}, b_{2} \in \mathcal{B} \text { and } a \in \mathcal{A} .
$$

For (2.3), we have

$$
\begin{aligned}
\left\langle\chi(\Delta(b)), a_{1} \otimes a_{2}\right\rangle & =\left(\Delta(b), \phi\left(a_{1}\right) \otimes \phi\left(a_{2}\right)\right)_{\mathcal{B}} \\
& =\left(\phi^{-1}(b), a_{1} a_{2}\right)_{\mathcal{A}} \\
& =\left(b, \phi\left(a_{1} a_{2}\right)\right)_{\mathcal{B}} \\
& =\left\langle\chi(b), a_{1} a_{2}\right\rangle .
\end{aligned}
$$

For (2.4), we have

$$
\begin{aligned}
\left\langle\chi\left(b_{1} b_{2}\right), a\right\rangle & =\left(b_{1} b_{2}, \phi(a)\right)_{\mathcal{B}} \\
& =\left(\phi^{-1}\left(b_{1}\right) \otimes \phi^{-1}\left(b_{2}\right), \Delta(a)\right)_{\mathcal{A}} \\
& =\sum_{a}\left(\phi^{-1}\left(b_{1}\right), a^{\prime}\right)_{\mathcal{A}}\left(\phi^{-1}\left(b_{2}\right), a^{\prime \prime}\right)_{\mathcal{A}} \\
& =\sum_{a}\left(b_{1}, \phi\left(a^{\prime}\right)\right)_{\mathcal{B}}\left(b_{2}, \phi\left(a^{\prime \prime}\right)\right)_{\mathcal{B}} \\
& =\sum_{a}\left\langle\chi\left(b_{1}\right), a^{\prime}\right\rangle\left\langle\chi\left(b_{2}\right), a^{\prime \prime}\right\rangle \\
& =\left\langle\chi\left(b_{1}\right) \otimes \chi\left(b_{2}\right), \Delta(a)\right\rangle .
\end{aligned}
$$

We note that it follows from this result that a graded connected locally finite Hopf algebra $\mathcal{A}$ is self-dual provided it admits an inner product $(\cdot, \cdot)$ such that

$$
\left(a_{1} \otimes a_{2}, \Delta\left(a_{3}\right)\right)=\left(a_{1} a_{2}, a_{3}\right)
$$

for all $a_{1}, a_{2}, a_{3} \in \mathcal{A}$. 


\section{Symmetric and Quasi-Symmetric Functions}

Let $\mathcal{B}$ be the subalgebra of the formal power series ring $k\left[\left[t_{1}, t_{2}, \ldots\right]\right]$ consisting of those formal power series of bounded degree, where each $t_{i}$ has degree 1. An element $f \in \mathcal{B}$ is called a symmetric function if the coefficients in $f$ of the monomials

$$
t_{n_{1}}^{i_{1}} t_{n_{2}}^{i_{2}} \cdots t_{n_{k}}^{i_{k}} \quad \text { and } \quad t_{1}^{i_{1}} t_{2}^{i_{2}} \cdots t_{k}^{i_{k}}
$$

agree for any sequence of distinct positive integers $n_{1}, n_{2}, \ldots, n_{k}$ : an element $f \in \mathcal{B}$ is called a quasi-symmetric function if the coefficients in $f$ of the monomials (3.1) agree for any strictly increasing sequence $n_{1}<n_{2}<\cdots<n_{k}$ of positive integers. The sets of symmetric and quasi-symmetric functions are denoted Sym and QSym respectively: both are subalgebras of $\mathcal{B}$, and evidently Sym $\subset$ QSym.

As a vector space, QSym is generated by the monomial quasi-symmetric functions $M_{I}$, which are indexed by compositions (finite sequences) of positive integers: for $I=\left(i_{1}, \ldots, i_{k}\right)$,

$$
M_{I}=\sum_{n_{1}<n_{2}<\cdots<n_{k}} t_{n_{1}}^{i_{1}} t_{n_{2}}^{i_{2}} \cdots t_{n_{k}}^{i_{k}} .
$$

If we forget order in a composition, we get a partition: a vector-space basis for Sym is given by the monomial symmetric functions

$$
m_{\lambda}=\sum_{\phi(I)=\lambda} M_{I},
$$

where $\phi$ is the function from compositions to partitions that forgets order. For example, $m_{2,1,1}=M_{(2,1,1)}+M_{(1,2,1)}+M_{(1,1,2)}$.

It is well-known that Sym, as an algebra, is freely generated by several sets of symmetric functions (see, e.g., [17]):

1. The elementary symmetric functions $e_{k}=m_{1^{k}}$ (where $1^{k}$ means 1 repeated $k$ times);

2. The complete symmetric functions

$$
h_{k}=\sum_{|\lambda|=k} m_{\lambda}=\sum_{|I|=k} M_{I}
$$

3. The power-sum symmetric functions $p_{k}=m_{k}$. 
There is a duality between the $e_{k}$ and the $h_{k}$, reflected in the (graded) identity

$$
\left(1+e_{1}+e_{2}+\cdots\right)\left(1-h_{1}+h_{2}-\cdots\right)=1
$$

There is also a well-known Hopf algebra structure on Sym [9]. This structure can be defined by making the elementary symmetric functions divided powers, i.e.,

$$
\Delta\left(e_{k}\right)=\sum_{i+j=k} e_{i} \otimes e_{j}
$$

Equivalently, the $h_{i}$ are required to be divided powers, or the $p_{i}$ primitives. For this Hopf algebra structure,

$$
\Delta\left(m_{\lambda}\right)=\sum_{\lambda=\mu \cup \nu} m_{\mu} \otimes m_{\nu}
$$

where the sum is over all pairs $(\mu, \nu)$ such that $\mu \cup \nu=\lambda$ as multisets. For example, $\Delta\left(m_{2,1,1}\right)$ is

$$
1 \otimes m_{2,1,1}+m_{1} \otimes m_{2,1}+m_{2} \otimes m_{1,1}+m_{1,1} \otimes m_{2}+m_{2,1} \otimes m_{1}+m_{2,1,1} \otimes 1 .
$$

The Hopf algebra Sym is commutative and cocommutative, so its antipode $S$ is an algebra isomorphism with $S^{2}=\mathrm{id}$. In fact, as follows from (3.2), $S\left(e_{i}\right)=(-1)^{i} h_{i}$. To see that Sym is self-dual, note that it admits an inner product $(\cdot, \cdot)$ such that

$$
\left(h_{\lambda}, m_{\mu}\right)=\delta_{\lambda, \mu}
$$

for all partitions $\lambda, \mu$ (where $h_{\lambda}$ means $h_{\lambda_{1}} h_{\lambda_{2}} \ldots$ for $\lambda=\lambda_{1}, \lambda_{2}, \ldots$ ) [17, $\S$ I.4]. Then by equation (3.3),

$$
\left(h_{\mu} h_{\nu}, m_{\lambda}\right)=\left(h_{\mu} \otimes h_{\nu}, \Delta\left(m_{\lambda}\right)\right)=\delta_{\mu \cup \nu, \lambda}
$$

so Sym is self-dual by Theorem 2.1 .

To give QSym the structure of a graded connected Hopf algebra, one defines a coproduct $\Delta$ by

$$
\Delta\left(M_{\left(p_{1}, \ldots, p_{k}\right)}\right)=\sum_{j=0}^{k} M_{\left(p_{1}, \ldots, p_{j}\right)} \otimes M_{\left(p_{j+1}, \ldots, p_{k}\right)} .
$$

This coproduct extends that on Sym, but it is no longer cocommutative: for example,

$$
\Delta\left(M_{(2,1,1)}\right)=1 \otimes M_{(2,1,1)}+M_{2} \otimes M_{(2,1)}+M_{(2,1)} \otimes M_{1}+M_{(2,1,1)} \otimes 1 .
$$


The antipode of QSym is given by [5, Prop. 3.4]

$$
S\left(M_{I}\right)=(-1)^{\ell(I)} \sum_{J \preceq I} M_{\bar{J}}
$$

where $\preceq$ is the refinement order on compositions and $\bar{I}$ is the reverse of $I$.

Since QSym is commutative but not cocommutative, it cannot be selfdual: in fact, its dual is the Hopf algebra NSym of noncommutative symmetric functions in the sense of Gelfand et al. [10. As an algebra NSym is the noncommutative polynomial algebra $k\left\langle E_{1}, E_{2}, \ldots\right\rangle$, with $E_{i}$ in degree $i$, and the Hopf algebra structure is determined by declaring the $E_{i}$ divided powers. There is an abelianization homomorphism $\tau$ : NSym $\rightarrow$ Sym sending $E_{i}$ to the elementary symmetric function $e_{i}$ : its dual $\tau^{*}:$ Sym $\rightarrow$ QSym is the inclusion Sym $\subset$ QSym.

\section{Hopf Algebras of Rooted Trees}

A rooted tree is a partially ordered set with a unique maximal element such that, for any element $v$, the set of elements exceeding $v$ in the partial order forms a chain. We call the elements of a rooted tree vertices, the maximal element the root, and the minimal elements leaves. If a vertex $v$ covers $w$ in the partial order, we call $v$ the parent of $w$ and $w$ a child of $v$. We visualize a rooted tree as a directed graph with an edge from each vertex to each of its children: the root (uniquely) has no incoming edges, and leaves have no outgoing edges. Let $\mathcal{T}$ be the set of rooted trees, and

$$
\mathcal{T}_{n}=\{t \in \mathcal{T}:|t|=n+1\}
$$

the set of rooted trees with $n+1$ vertices. There is a graded vector space

$$
k \mathcal{T}=\bigoplus_{n \geq 0} k \mathcal{T}_{n}
$$

with the set of rooted trees as basis.

Each rooted tree $t$ has a symmetry group $\operatorname{Symm}(t)$, the group of automorphisms of $t$ as a poset (or directed graph). This group can be explicitly described as follows. For each vertex $v$ of a rooted tree $t$, let $t_{v}$ be the rooted tree consisting of $v$ and its descendants (with the partial order inherited from $t$ ). If the set of children of $v$ is $C(v)=\left\{v_{1}, \ldots, v_{k}\right\}$, let $S G(t, v)$ be the group 
of permutations of $C(v)$ generated by those that exchange $v_{i}$ with $v_{j}$ when $t_{v_{i}}$ and $t_{v_{j}}$ are isomorphic rooted trees. Then

$$
\operatorname{Symm}(t)=\prod_{\text {vertices } v \text { of } t} S G(t, v) .
$$

By a forest we mean a monomial in rooted trees, with the rooted trees thought of as commuting with each other. There is an algebra of forests, which is just the symmetric algebra $S(k \mathcal{T})$ over $k \mathcal{T}$ : the multiplication can be thought of as juxtaposition of forests. For any forest $t_{1} t_{2} \cdots t_{k}$, there is a rooted tree $B_{+}\left(t_{1} t_{2} \cdots t_{k}\right)$ given by attaching a new root vertex to each of the roots of $t_{1}, t_{2}, \ldots, t_{k}$, e.g.,

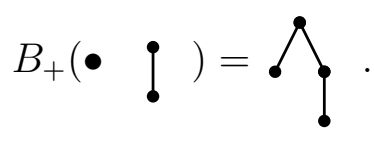

Also, let $B_{+}$send $1 \in S^{0}(k \mathcal{T})$ (thought of as the empty forest) to $\bullet \in \mathcal{T}_{0}$. If we grade $S(k \mathcal{T})$ by

$$
\left|t_{1} \cdots t_{k}\right|=\left|t_{1}\right|+\cdots+\left|t_{k}\right|
$$

where $|t|$ is the number of vertices of the rooted tree $t$, then $B_{+}: S(k \mathcal{T}) \rightarrow k \mathcal{T}$ is an isomorphism of graded vector spaces.

There is a product $\circ$ on $k \mathcal{T}$ defined by Grossman and Larson [13]. Given rooted trees $t$ and $t^{\prime}$, let $t=B_{+}\left(t_{1} t_{2} \cdots t_{n}\right)$ and $\left|t^{\prime}\right|=m$. Then $t \circ t^{\prime}$ is the sum of the $m^{n}$ rooted trees obtained by attaching each of the $t_{i}$ to a vertex of $t^{\prime}$ : if $t=\bullet$, set $t \circ t^{\prime}=t^{\prime}$. For example,

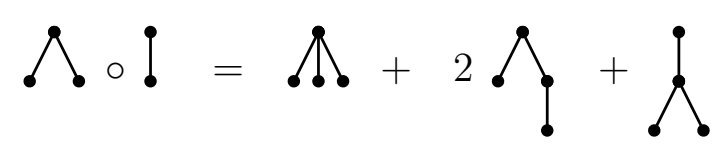

while

$$
\mathfrak{\jmath} \circ=\widehat{\jmath}+2 \widehat{\jmath} .
$$

This noncommutative product makes $k \mathcal{T}$ a graded algebra with two-sided unit $\bullet$. There is a coproduct $\Delta$ on $k \mathcal{T}$ defined by $\Delta(\bullet)=\bullet \otimes \bullet$ and

$$
\Delta\left(B_{+}\left(t_{1} t_{2} \cdots t_{k}\right)\right)=\sum_{I \cup J=\{1,2, \ldots, k\}} B_{+}(t(I)) \otimes B_{+}(t(J)),
$$


where $t_{1}, \ldots, t_{k}$ are rooted trees, the sum is over all disjoint pairs $(I, J)$ of subsets of $\{1,2, \ldots, k\}$ such that $I \cup J=\{1,2, \ldots, k\}$, and $t(I)$ means the product of $t_{i}$ for $i \in I$ (with the convention $B_{+}(t(\emptyset))=\bullet$ ). As is proved in [13], the vector space $k \mathcal{T}$ with product $\circ$ and coproduct $\Delta$ is a graded connected Hopf algebra.

The Connes-Kreimer Hopf algebra $\mathcal{H}_{K}$ is generated as a commutative algebra by the rooted trees. As a graded algebra, $\mathcal{H}_{K}$ is $S(k \mathcal{T})$ with the grading discussed above. The coproduct on $\mathcal{H}_{K}$ can be described recursively by setting $\Delta(1)=1 \otimes 1$ and

$$
\Delta(t)=t \otimes 1+\left(\mathrm{id} \otimes B_{+}\right) \Delta\left(B_{-}(t)\right),
$$

for rooted trees $t$, where $B_{-}$is the inverse of $B_{+}$and it is assumed that $\Delta$ acts multiplicatively on products of rooted trees.

Alternatively, $\Delta$ can be described on rooted trees $t$ by the formula

$$
\Delta(t)=t \otimes 1+\sum_{\text {admissible cuts } c} P^{c}(t) \otimes R^{c}(t) .
$$

Here a cut of a rooted tree $t$ is a subset of the edges of $t$, and a cut $c$ is admissible if any path from the root to a leaf meets $c$ at most once. If all the edges in $c$ are removed from $t$, then $t$ falls apart into smaller rooted trees: $R^{c}(t)$ is the component containing the original root, and $P^{c}(t)$ is the forest consisting of the rest of the components. We then extend $\Delta$ to forests by assuming it acts multiplicatively.

There is also a nice formula for the antipode of $\mathcal{H}_{K}$ in terms of cuts: for a rooted tree $t$,

$$
S(t)=-\sum_{\text {all cuts } c}(-1)^{|c|} P^{c}(t) R^{c}(t)
$$

where the sum is over all cuts $c$, and $|c|$ is the number of edges in $c$. Equation (4.4) can be proved by induction on $|t|$.

It follows from equation (4.3) that the "ladders" $\ell_{i}$ (where $\ell_{i}$ is the unbranched tree with $i$ vertices) are divided powers. Thus, there is a Hopf algebra homomorphism $\phi: \operatorname{Sym} \rightarrow \mathcal{H}_{K}$ sending $e_{i}$ to $\ell_{i}$.

We note that, for a forest $u$ and rooted tree $t$, the rooted tree $t^{\prime}$ can only appear in $B_{+}(u) \circ t^{\prime}$ if there is an admissible cut $c$ of $t^{\prime}$ such that

$$
P^{c}\left(t^{\prime}\right)=u \quad \text { and } \quad R^{c}\left(t^{\prime}\right)=t .
$$


Generalizing the definition of [14, §4], we define

$$
n\left(u, t ; t^{\prime}\right)=\text { the number of times } t^{\prime} \text { appears in } B_{+}(u) \circ t
$$

and

$m\left(u, t ; t^{\prime}\right)=$ the number of distinct admissible cuts $c$ for which (4.5) holds.

Lemma 4.1. For $u, t, t^{\prime}$ as above,

$$
n\left(u, t ; t^{\prime}\right)\left|\operatorname{Symm}\left(t^{\prime}\right)\right|=m\left(u, t ; t^{\prime}\right)\left|\operatorname{Symm}\left(B_{+}(u)\right)\right||\operatorname{Symm}(t)| .
$$

Proof. This is a slight extension of the proof of [14, Prop. 4.3]. First, let $u=s_{1} s_{2} \cdots s_{n}$ for rooted trees $s_{i}$ : then

$$
\operatorname{Symm}\left(B_{+}(u)\right)=P \times \prod_{i=1}^{n} \operatorname{Symm}\left(s_{i}\right),
$$

where $P$ is the group that permutes those $s_{i}$ that are isomorphic. Suppose (4.5) holds: let Fix $\left(c, t^{\prime}\right)$ be the subgroup of $\operatorname{Symm}\left(t^{\prime}\right)$ that holds all the edges of $c$ and everything "below" them pointwise fixed, and $Q$ the subgroup of $P$ that permutes identical parts of $u$ that are attached to the same vertex in $t^{\prime}$. Then $m\left(u, t ; t^{\prime}\right)$ is the cardinality of the orbit of $c$ under $\operatorname{Symm}\left(t^{\prime}\right)$, which is

$$
\operatorname{Symm}\left(t^{\prime}\right) / \operatorname{Fix}\left(c, t^{\prime}\right) \times \prod_{i=1}^{n} \operatorname{Symm}\left(s_{i}\right) \times Q,
$$

and so

$$
m\left(u, t ; t^{\prime}\right)=\frac{\left|\operatorname{Symm}\left(t^{\prime}\right)\right|}{\left|\operatorname{Fix}\left(c, t^{\prime}\right)\right||Q| \prod_{i=1}^{n}\left|\operatorname{Symm}\left(s_{i}\right)\right|} .
$$

On the other hand, if we think of attaching the parts of $u$ to the rooted tree $t$, we see that

$$
n\left(u, t ; t^{\prime}\right)=\frac{|P||\operatorname{Symm}(t)|}{|Q|\left|\operatorname{Fix}\left(c, t^{\prime}\right)\right|}
$$

and the conclusion follows.

Using the lemma, we can prove that the Hopf algebras $\mathcal{H}_{K}$ and $k \mathcal{T}$ are dual to each other: for a somewhat different proof, see [14, Prop. 4.4].

Theorem 4.2. The Hopf algebra $k \mathcal{T}$ is the graded dual of $\mathcal{H}_{K}$. 
Proof. First note that there is an inner product on $k \mathcal{T}$ defined by

$$
\left(t, t^{\prime}\right)= \begin{cases}|\operatorname{Symm}(t)|, & \text { if } t^{\prime}=t \\ 0, & \text { otherwise }\end{cases}
$$

This inner product extends to $S(k \mathcal{T})=\mathcal{H}_{K}$ via $(u, v)=\left(B_{+}(u), B_{+}(v)\right)$ (since $\operatorname{Symm}\left(B_{+}(t)\right) \cong \operatorname{Symm}(t)$, this definition is consistent). So if we use Theorem 2.1 with $\phi=B_{+}$, hypothesis (a) of the theorem is satisfied. Hypothesis (b) follows easily from definitions, so it remains to prove

$$
(u \otimes v, \Delta(w))=\left(B_{+}(u) \circ B_{+}(v), B_{+}(w)\right)
$$

for monomials $u, v$, and $w$ of $\mathcal{H}_{K}$. Writing $t_{1}=B_{+}(u), t_{2}=B_{+}(v)$ and $t_{3}=B_{+}(w)$, equation (4.7) is

$$
\left(B_{-}\left(t_{1}\right) \otimes B_{-}\left(t_{2}\right), \Delta\left(B_{-}\left(t_{3}\right)\right)\right)=\left(t_{1} \circ t_{2}, t_{3}\right),
$$

which in turn, by using equation (4.2), is

$$
\left(u \otimes t_{2}, \Delta\left(t_{3}\right)-t_{3} \otimes 1\right)=\left(t_{1} \circ t_{2}, t_{3}\right) .
$$

Both sides of equation (4.8) are nonzero if and only if there is an admissible cut $c$ of $t_{3}$ such that

$$
P^{c}\left(t_{3}\right)=u \quad \text { and } \quad R^{c}\left(t_{3}\right)=t_{2}
$$

in which case it is

$$
m\left(u, t_{2} ; t_{3}\right)\left|\operatorname{Symm}\left(t_{1}\right)\right|\left|\operatorname{Symm}\left(t_{2}\right)\right|=n\left(u, t_{2} ; t_{3}\right)\left|\operatorname{Symm}\left(t_{3}\right)\right|,
$$

i.e., the lemma above.

\section{$5 \quad$ Hopf Algebras of Planar Rooted Trees}

In parallel to the preceding section, we define $\mathcal{P}$ to be the graded poset of planar rooted trees, and $k \mathcal{P}$ the corresponding graded vector space. A planar rooted tree is a particular realization of a rooted tree in the plane, so we consider

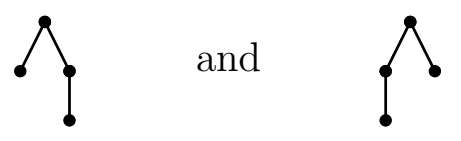


as distinct planar rooted trees. The tensor algebra $T(k \mathcal{P})$ can be regarded as the algebra of ordered forests of planar rooted trees, and there is a linear map $B_{+}: T(k \mathcal{P}) \rightarrow k \mathcal{P}$ that makes a planar rooted tree out of an ordered forest of planar rooted trees by attaching a new root vertex. With the same conventions about grading as in the previous section, $B_{+}$is an isomorphism of graded vector spaces.

Planar rooted trees with $n$ non-root vertices correspond to balanced bracket arrangements (BBAs) of weight $n$, i.e., arrangements of the symbols $\langle$ and $\rangle$ such that

1. the symbol $<$ and the symbol $\rangle$ each occur $n$ times, and

2. reading left to right, the count of 〈's never falls behind the count of 〉's. For example, the five BBAs of weight 3, to wit

$$
\langle\langle\langle\rangle\rangle\rangle, \quad\langle\langle\rangle\langle\rangle\rangle, \quad\langle\rangle\langle\langle\rangle\rangle, \quad\langle\langle\rangle\rangle\langle\rangle, \quad \text { and } \quad\langle\rangle\langle\rangle\langle\rangle,
$$

correspond respectively to

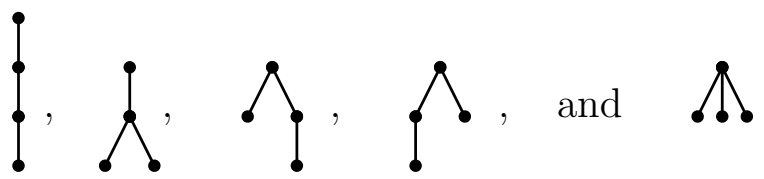

in $\mathcal{P}_{3}$. Note that the empty BBA corresponds to the 1 -vertex tree $\bullet$. This representation is similar to that of Holtkamp [15], but differs in that our BBAs are not necessarily irreducible (see the next paragraph): to go from Holtkamp's representation to ours, remove the outermost pair of brackets. It is well-known that the number of BBAs of weight $n$ is the $n$th Catalan number

$$
C_{n}=\frac{1}{n+1}\left(\begin{array}{c}
2 n \\
n
\end{array}\right)
$$

We call a BBA $c$ irreducible if $c=\left\langle c^{\prime}\right\rangle$ for some BBA $c^{\prime}$. If a BBA is not irreducible, it can be written as a juxtaposition $c_{1} c_{2} \cdots c_{k}$ of irreducible BBAs, which we call the components of $c$. The components of a BBA correspond to the branches of the root in the associated planar rooted tree.

We define a product on $k \mathcal{P}$ via the representation in terms of BBAs. If the planar rooted trees $T$ and $T^{\prime}$ are represented by BBAs $c$ and $c^{\prime}$ respectively, let $c_{1} c_{2} \cdots c_{k}$ be the components of $c$. Then $T \circ T^{\prime}$ is the sum of planar rooted trees corresponding to the asymmetric shuffle product of $c$ with $c^{\prime}$, i.e., the 
sum of the BBAs obtained by shuffling the symbols $c_{1} c_{2} \cdots c_{k}$ into the BBA $c^{\prime}$. For example, if $c=c_{1} c_{2}$ and $c^{\prime}=\langle\rangle$ then the asymmetric shuffle product $c \sqcup c^{\prime}$ is

$$
c_{1} c_{2}\langle\rangle+c_{1}\left\langle c_{2}\right\rangle+c_{1}\langle\rangle c_{2}+\left\langle c_{1} c_{2}\right\rangle+\left\langle c_{1}\right\rangle c_{2}+\langle\rangle c_{1} c_{2}
$$

If $c_{1}=c_{2}=\langle\rangle$, this reduces to

$$
\langle\rangle\langle\rangle \omega\langle\rangle=3\langle\rangle\langle\rangle\langle\rangle+\langle\rangle\langle\langle\rangle\rangle+\langle\langle\rangle\rangle\langle\rangle+\langle\langle\rangle\langle\rangle\rangle,
$$

and hence

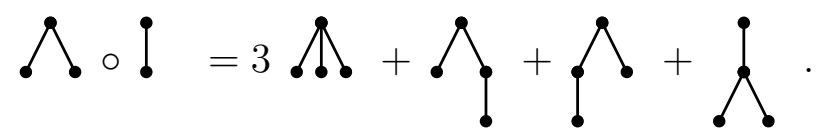

On the other hand, shuffling a single component $c$ into \langle\rangle\langle\rangle gives

$$
c\langle\rangle\langle\rangle+\langle c\rangle\langle\rangle+\langle\rangle c\langle\rangle+\langle\rangle\langle c\rangle+\langle\rangle\langle\rangle c
$$

which for $c=\langle\rangle$ reduces to

$$
\langle\rangle \sqcup\langle\rangle\langle\rangle=3\langle\rangle\langle\rangle\langle\rangle+\langle\rangle\langle\langle\rangle\rangle+\langle\langle\rangle\rangle\langle\rangle
$$

Thus

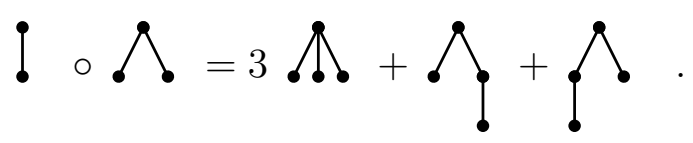

Now we make $k \mathcal{P}$ a coalgebra by defining a coproduct $\Delta$ on BBAs by

$$
\Delta(c)=\sum_{i=0}^{k} c_{1} \cdots c_{i} \otimes c_{i+1} \cdots c_{k}
$$

where $c=c_{1} c_{2} \cdots c_{k}$ is the decomposition of $c$ into irreducible components.

Theorem 5.1. The product $\circ$ and coproduct $\Delta$ make $k \mathcal{P}$ a graded connected Hopf algebra. 
Proof. There are two main items to check: the associativity of o, and the multiplicativity of $\Delta$. We use the representation of planar rooted trees by BBAs as outlined above. For BBAs $a$ and $b$, each term of $a \sqcup b$ has components that are either components of $a$, or components of $b$ into which some components of a may be inserted: and the order of the components among those of $a$ and among those of $b$ is preserved. Thus each component of a term of $(a \sqcup b) \sqcup c$ is a component of $a$, a component of $b$ into which some components of $a$ may be inserted, or a component of $c$ into which components of $a$ and components of $b$ (possibly including some components of $a$ ) may be inserted: and the order of components among those of $a, b$, and $c$ is preserved. But terms of $a \amalg(b \sqcup c)$ can be described the same way.

For multiplicativity, let $a, b$ be BBAs, with decomposition into components $a=a_{1} \cdots a_{n}$ and $b=b_{1} \cdots b_{m}$. Then each term $c^{\prime} \otimes c^{\prime \prime}$ of $\Delta(a \sqcup b)$ comes from the term

$$
\left(a_{1} \cdots a_{i} \sqcup b_{1} \cdots b_{j}\right) \otimes\left(a_{i+1} \cdots a_{n} \sqcup b_{j+1} \cdots b_{m}\right)
$$

of $\Delta(a) \sqcup \Delta(b)$, where $i, j$ are the largest integers such that the components $a_{i}$ and $b_{j}$ respectively occur in $c^{\prime}$.

The Foissy Hopf algebra $\mathcal{H}_{F}$ is defined as follows. As an algebra, it is the tensor algebra $T(k \mathcal{P})$. The coalgebra structure can be defined by the same equation (4.3) as for $\mathcal{H}_{K}$, except that rooted trees are replaced by planar rooted trees, and the forests are ordered. (We remark that there is a natural order on the vertices of a planar rooted tree, which means that for a cut $c$ of a planar rooted tree $T$ the forest $P^{c}(T)$ has a natural ordering.)

Alternatively, the coalgebra structure on $\mathcal{H}_{F}$ can be defined by

$$
\Delta(F)=\sum_{F^{\prime} \subseteq F}\left(F-F^{\prime}\right) \otimes F^{\prime}
$$

where the sum is over all rooted subforests $F^{\prime}$ of $F$ : if $F=T_{1} T_{2} \cdots T_{n}$, then a rooted subforest $F^{\prime}$ of $F$ is a forest $T_{1}^{\prime} T_{2}^{\prime} \cdots T_{n}^{\prime}$ such that each $T_{i}^{\prime}$ is either a subtree of $T_{i}$ that contains the root, or empty. For such $F$ and $F^{\prime}$, set $F-F^{\prime}=F_{1} F_{2} \cdots F_{n}$, where

$$
F_{i}= \begin{cases}P^{c}\left(T_{i}\right), & \text { if } T_{i}^{\prime} \neq \emptyset \text { and } R^{c}\left(T_{i}\right)=T_{i}^{\prime}, \\ T_{i}, & \text { if } T_{i}^{\prime}=\emptyset\end{cases}
$$


The equation (4.4) for the antipode in $\mathcal{H}_{K}$ almost works in $\mathcal{H}_{F}$, but must be slightly modified. For a planar rooted tree $T$,

$$
S(T)=-\sum_{\text {all cuts } c \text { of } T}(-1)^{|c|} \overline{P^{c}(T)} R^{c}(T),
$$

where $\bar{F}$ denotes the reverse of the ordered forest $F$ (cf. [7, Théorème 44]). Note that $S$ is an antiautomorphism of the noncommutative algebra $\mathcal{H}_{F}$, and $S^{2} \neq \mathrm{id}$.

Theorem 5.2. The Hopf algebra $(k \mathcal{P}, \circ, \Delta)$ is dual to the Foissy Hopf algebra $\mathcal{H}_{F}$.

Proof. As in the preceding section, this boils down to the identity

$$
(u \otimes v, \Delta(w))=\left(B_{+}(u) \circ B_{+}(v), B_{+}(w)\right),
$$

where now the inner product is defined by $\left(T, T^{\prime}\right)=\delta_{T, T^{\prime}}$. The proof is essentially the same as that for Theorem 4.2 above, but much easier since there are no symmetry groups to complicate things: for planar rooted trees $T, T^{\prime}$ and an ordered forest $F$ of planar rooted trees, $\left(B_{+}(F) \circ T, T^{\prime}\right)$ is both the multiplicity of $T^{\prime}$ in $B_{+}(F) \circ T$ and the number of cuts of $T^{\prime}$ with $P^{c}\left(T^{\prime}\right)=$ $F$ and $R^{c}\left(T^{\prime}\right)=T$.

Theorem 5.3. $\mathcal{H}_{F}$ is self-dual.

Proof. This follows from the existence of an inner product $(\cdot, \cdot)_{F}$ on $\mathcal{H}_{F}$ with

$$
\left(F_{1} F_{2}, F_{3}\right)_{F}=\left(F_{1} \otimes F_{2}, \Delta\left(F_{3}\right)\right)_{F}
$$

for ordered forests $F_{1}, F_{2}, F_{3}$. Such an inner product is constructed in [7, $\S 6]$.

\section{$6 \quad$ Lifting to the Foissy Hopf Algebra}

The "ladder" trees $\ell_{i}$ can be thought of as planar rooted trees, and since they are divided powers in $\mathcal{H}_{F}$ there is a Hopf algebra homomorphism $\Phi$ : $\mathrm{NSym} \rightarrow \mathcal{H}_{F}$ sending $E_{i}$ to $\ell_{i}$. In fact, there is a commutative diagram of Hopf algebras

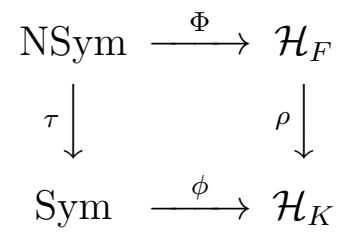


where the map $\rho: \mathcal{H}_{F} \rightarrow \mathcal{H}_{K}$ sends each planar rooted tree to the corresponding rooted tree, and forgets order in products. In the commutative diagram dual to (6.1), i.e.,

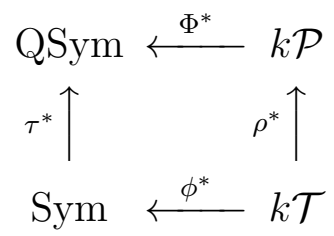

the maps can be described explicitly as follows. As noted earlier, $\tau^{*}$ is the inclusion. For a partition $\lambda=\lambda_{1}, \lambda_{2}, \ldots, \lambda_{k}$, let

$$
t_{\lambda}=B_{+}\left(\ell_{\lambda_{1}} \ell_{\lambda_{2}} \cdots \ell_{\lambda_{k}}\right) \in \mathcal{T}
$$

Then for rooted trees $t$,

$$
\phi^{*}(t)= \begin{cases}\left|\operatorname{Symm}\left(t_{\lambda}\right)\right| m_{\lambda}, & \text { if } t=t_{\lambda} \text { for some partition } \lambda \\ 0, & \text { otherwise. }\end{cases}
$$

Of course

$$
\left|\operatorname{Symm}\left(t_{\lambda}\right)\right|=m_{1}(\lambda) ! m_{2}(\lambda) ! \cdots,
$$

where $m_{i}(\lambda)$ is the multiplicity of $i$ in $\lambda$. The formula "upstairs" is simpler: if for a composition $I=\left(i_{1}, i_{2}, \ldots, i_{k}\right)$ we define the planar rooted tree

$$
T_{I}=B_{+}\left(\ell_{i_{1}} \ell_{i_{2}} \cdots \ell_{i_{k}}\right) \in \mathcal{P},
$$

then

$$
\Phi^{*}(T)= \begin{cases}M_{I}, & \text { if } T=T_{I} \text { for some composition } I \\ 0, & \text { otherwise. }\end{cases}
$$

For a rooted tree $t$,

$$
\rho^{*}(t)=|\operatorname{Symm}(t)| \sum_{T \in \rho^{-1}(t)} T
$$

\subsection{Some Particular Families of Rooted Trees}

It is often easier to establish properties of rooted trees by working "upstairs" in diagram (6.2) rather than directly. For example, the elements

$$
\kappa_{n}=\sum_{t \in \mathcal{T}_{n}} \frac{t}{|\operatorname{Symm}(t)|} \in k \mathcal{T}
$$


are most easily understood by considering their images

$$
\rho^{*}\left(\kappa_{n}\right)=\sum_{T \in \mathcal{P}_{n}} T \in k \mathcal{P} .
$$

In this way it can be seen easily that the $\kappa_{n}$ form a set of divided powers, and that $\phi^{*}\left(\kappa_{n}\right)=h_{n}$. Recalling the identity (3.2) in Sym, define elements $\epsilon_{n}$ of $k \mathcal{T}$ inductively by

$$
\epsilon_{n}=\kappa_{1} \circ \epsilon_{n-1}-\kappa_{2} \circ \epsilon_{n-2}+\cdots+(-1)^{n-1} \kappa_{n}, \quad \epsilon_{0}=\bullet .
$$

Proposition 6.1. The elements $\epsilon_{n}$ satisfy

(a) $\epsilon_{n}=(-1)^{n} S\left(\kappa_{n}\right)$

(b) $\phi^{*}\left(\epsilon_{n}\right)=e_{n}$

(c) $n ! \epsilon_{n}=t_{1^{n}}$, where $1^{n}$ is a string of $n$ ones.

Proof. Part (a) follows from equation (6.4), and then part (b) follows by applying $\phi^{*}$. To prove part (c), recall from Theorem 4.2 that

$$
\langle\chi(t), F\rangle=\left(t, B_{+}(F)\right)
$$

where $(\cdot, \cdot)$ is the inner product given by (4.6). Hence $\chi\left(\kappa_{n}\right)$ is the linear functional on $\mathcal{H}_{K}$ that sends every forest of degree $n$ to 1 (and all other forests to 0 ). If $F$ is a forest of weight $n$, it follows from equation (4.4) that $\chi\left(\kappa_{n}\right)$ is zero on any forest $S(F)$ except

$$
F=\bullet \bullet \cdots \bullet,
$$

on which it is $(-1)^{n}$. Hence

$$
S\left(\kappa_{n}\right)=(-1)^{n} \frac{B_{+}(\bullet \bullet \cdots \bullet)}{\left|\operatorname{Symm}\left(B_{+}(\bullet \bullet \cdots \bullet)\right)\right|}=(-1)^{n} \frac{t_{1^{n}}}{n !} .
$$

Zhao [18] defines a homomorphism NSym $\rightarrow k \mathcal{T}$ sending $E_{n}$ to $\epsilon_{n}$; in view of the preceding result, it sends the noncommutative analogue $(-1)^{n} S\left(E_{n}\right)$ of the $n$th complete symmetric function to $\kappa_{n}$. There are several distinct analogues of the power-sum symmetric functions in NSym (see [10]): their 
images in $k \mathcal{T}$ under Zhao's homomorphism are described in [18, Theorem 4.6].

If we define an operator $\mathfrak{N}: k \mathcal{T} \rightarrow k \mathcal{T}$ by $\mathfrak{N}(t)=\ell_{2} \circ t$, then

$$
\mathfrak{N}^{k}(t)=\sum_{\left|t^{\prime}\right|=|t|+k} n\left(t ; t^{\prime}\right) t^{\prime}
$$

for some coefficients $n\left(t ; t^{\prime}\right)$. Apply $\phi^{*}$ to get

$$
e_{1}^{k} \phi^{*}(t)=\sum_{\left|t^{\prime}\right|=|t|+k} n\left(t ; t^{\prime}\right) \phi^{*}\left(t^{\prime}\right)
$$

Two special cases of this equation are of some interest. First, let $k=1$ : then $n\left(t ; t^{\prime}\right)=n\left(\bullet, t ; t^{\prime}\right)$ as defined in $\S 4$ and equation (6.5) implies

$$
e_{1} \phi^{*}\left(t_{\lambda}\right)=\sum_{|\mu|=|\lambda|+1} n\left(\bullet, t_{\lambda} ; t_{\mu}\right) \phi^{*}\left(t_{\mu}\right)
$$

for all partitions $\lambda$. Hence, using (6.3) and Lemma 4.1,

$$
m\left(\bullet, t_{\lambda} ; t_{\mu}\right)=\frac{\left|\operatorname{Symm}\left(t_{\mu}\right)\right|}{\left|\operatorname{Symm}\left(t_{\lambda}\right)\right|} n\left(\bullet, t_{\lambda} ; t_{\mu}\right)=\text { coefficient of } m_{\mu} \text { in } e_{1} m_{\lambda} .
$$

Second, suppose $t=\bullet$. Then equation $(6.5)$ is

$$
e_{1}^{k}=\sum_{|\lambda|=k} n\left(\bullet ; t_{\lambda}\right) \phi^{*}\left(t_{\lambda}\right)
$$

which, compared with

$$
e_{1}^{k}=\sum_{|\lambda|=k}\left(\begin{array}{l}
k \\
\lambda
\end{array}\right) m_{\lambda}
$$

gives a formula for $n\left(\bullet ; t_{\lambda}\right)$ (cf. equation (1) of [2]):

$$
n\left(\bullet ; t_{\lambda}\right)=\frac{1}{\left|\operatorname{Symm}\left(t_{\lambda}\right)\right|}\left(\begin{array}{c}
|\lambda| \\
\lambda
\end{array}\right) .
$$




\subsection{Combinatorial Dyson-Schwinger equations}

We now illustrate the use of the map $\rho$ in diagram (6.1) to solve the combinatorial Dyson-Schwinger equation

$$
X=1+B_{+}\left(X^{p}\right)
$$

where $X$ is a formal sum of elements

$$
X=1+x_{1}+x_{2}+\cdots
$$

of $\mathcal{H}_{K}$, with $x_{i}$ of degree $i$, and $p$ is a real number. If we write $\bar{X}=x_{1}+x_{2}+$ $\cdots$, equation (6.6) is

$$
\bar{X}=B_{+}\left((1+\bar{X})^{p}\right)=B_{+}\left(1+\left(\begin{array}{l}
p \\
1
\end{array}\right) \bar{X}+\left(\begin{array}{l}
p \\
2
\end{array}\right) \bar{X}^{2}+\cdots\right)
$$

where

$$
\left(\begin{array}{l}
p \\
k
\end{array}\right)=\frac{p(p-1) \cdots(p-k+1)}{k !}
$$

Then

$$
x_{n+1}=B_{+}\left(\left\{1+\left(\begin{array}{l}
p \\
1
\end{array}\right) \bar{X}+\left(\begin{array}{l}
p \\
2
\end{array}\right) \bar{X}^{2}+\cdots\right\}_{n}\right),
$$

where $\{\cdot\}_{n}$ means degree- $n$ part. Consider (6.6) as an equation in $\mathcal{H}_{F}$ : if

$$
1+X_{1}+X_{2}+\cdots=1+\tilde{X}
$$

is its solution, then $X_{1}=B_{+}(1)=\bullet$, and

$$
X_{n+1}=B_{+}\left(\left(\begin{array}{l}
p \\
1
\end{array}\right)\{\tilde{X}\}_{n}+\left(\begin{array}{l}
p \\
2
\end{array}\right)\left\{(\tilde{X})^{2}\right\}_{n}+\cdots\right)
$$

for $n \geq 1$. Thus

$$
X_{n+1}=\sum_{k \leq n}\left(\begin{array}{l}
p \\
k
\end{array}\right) B_{+}\left(\sum_{n_{1}+\cdots+n_{k}=n} X_{n_{1}} \cdots X_{n_{k}}\right),
$$

where the inner sum is over length- $k$ compositions of $n$. We claim that equation (6.8) has the solution

$$
X_{n}=\sum_{T \in \mathcal{P}_{n-1}} C_{p}(T) T
$$


where $C_{p}(T)$ is defined as follows. For a vertex $v$ of a planar rooted tree $T$, let $c(v)$ be the number of children of $v$. Let $\bar{V}(T)$ be the set of vertices of $T$ with $c(v) \neq 0$ : then

$$
C_{p}(T)=\prod_{v \in \bar{V}(T)}\left(\begin{array}{c}
p \\
c(v)
\end{array}\right) .
$$

For example,

$$
C_{p}(\widehat{\jmath})=\left(\begin{array}{l}
p \\
2
\end{array}\right) p .
$$

To see that (6.9) really does solve equation (6.8), we use induction on $n$. Suppose equation (6.9) holds through dimension $n$, and consider the coefficient of $T$ in $X_{n+1}$ for $T \in \mathcal{P}_{n}$. Now $T$ has a unique expression as $B_{+}\left(T_{1} T_{2} \cdots T_{k}\right)$, where $T_{1} T_{2} \cdots T_{k}$ is an ordered forest of planar rooted trees such that

$$
\left|T_{1}\right|+\left|T_{2}\right|+\cdots+\left|T_{k}\right|=n .
$$

From equation (6.8), we see that the only contribution to the coefficient of $T$ can come from $X_{n_{1}} X_{n_{2}} \cdots X_{n_{k}}$, for $n_{k}=\left|T_{k}\right|$. By the induction hypothesis, the coefficient of $T$ coming from equation (6.8) is

$$
\left(\begin{array}{l}
p \\
k
\end{array}\right) C_{p}\left(T_{1}\right) C_{p}\left(T_{2}\right) \cdots C_{p}\left(T_{k}\right):
$$

but this is evidently $C_{p}(T)$.

Now equation (6.10) makes sense for rooted trees $t$, and indeed for any planar rooted tree $T$ we have $C_{p}(T)=C_{p}(\rho(T))$. Projecting $\tilde{X}$ to $\bar{X}$ via $\rho$ gives the following result.

Theorem 6.2. The solution (6.7) of the combinatorial Dyson-Schwinger equation (6.6) in $\mathcal{H}_{K}$ is

$$
x_{n}=\sum_{t \in \mathcal{T}_{n-1}} e(t) C_{p}(t) t
$$

where $e(t)$ is the number of planar rooted trees $T$ such that $\rho(T)=t$. 
To compare our result with that of [1, Lemma 4], note that

$$
e(t)=\frac{1}{|\operatorname{Symm}(t)|} \prod_{v \in \bar{V}(t)} c(v) !
$$

so the coefficient in $x_{n}$ of a rooted tree $t$ is

$$
\frac{1}{|\operatorname{Symm}(t)|} \prod_{v \in \bar{V}(t)} c(v) !\left(\begin{array}{c}
p \\
c(v)
\end{array}\right)=\frac{1}{|\operatorname{Symm}(t)|} \prod_{v \in \bar{V}(t)} p(p-1) \cdots(p-c(v)+1) .
$$

The subalgebra of $\mathcal{H}_{K}$ generated by the $x_{n}$ is in fact a sub-Hopf-algebra of $\mathcal{H}_{K}$. This follows from our final result, which gives an explicit formula for $\Delta\left(x_{n}\right)$ in terms of the $x_{i}$.

Theorem 6.3. For the homogeneous parts $x_{n}$ of the solution (6.7) of the combinatorial Dyson-Schwinger equation in $\mathcal{H}_{K}$,

$$
\Delta\left(x_{n}\right)=x_{n} \otimes 1+\sum_{k=1}^{n} q_{n, k}\left(x_{1}, x_{2}, \ldots\right) \otimes x_{k}
$$

where $q_{n, n}=1$ and

$$
q_{n, k}\left(x_{1}, x_{2}, \ldots\right)=\sum_{i_{1}+2 i_{2}+\cdots=n-k}\left(\begin{array}{c}
k(p-1)+1 \\
i_{1}+i_{2}+\cdots
\end{array}\right) \frac{\left(i_{1}+i_{2}+\cdots\right) !}{i_{1} ! i_{2} ! \cdots} x_{1}^{i_{1}} x_{2}^{i_{2}} \cdots
$$

for $1 \leq k<n$.

Proof. We again work in $\mathcal{H}_{F}$ and project down to $\mathcal{H}_{K}$ via $\rho$. Equation (6.11) will follow from

$$
\Delta\left(X_{n}\right)=X_{n} \otimes 1+\sum_{k=1}^{n} Q_{n, k}\left(X_{1}, X_{2}, \ldots\right) \otimes X_{k}
$$

where $Q_{n, n}=1$ and

$$
Q_{n, k}\left(X_{1}, X_{2}, \ldots\right)=\sum_{q=1}^{n-k} \sum_{n_{1}+\cdots+n_{q}=n-k}\left(\begin{array}{c}
k(p-1)+1 \\
q
\end{array}\right) X_{n_{1}} \cdots X_{n_{q}}
$$


for $1 \leq k<n$. To prove equation (6.12), we use the equation (4.3) for the coproduct in $\mathcal{H}_{F}$. Fix positive integers $k \leq n$ and consider those terms in $\Delta\left(X_{n}\right)$ that contribute to the term

$$
C_{p}\left(T_{1}\right) T_{1} \cdots C_{p}\left(T_{q}\right) T_{q} \otimes C_{p}\left(T^{\prime}\right) T^{\prime} \quad \text { in } \quad X_{\left|T_{1}\right|} X_{\left|T_{2}\right|} \cdots X_{\left|T_{q}\right|} \otimes X_{k}
$$

for particular planar rooted trees $T_{1}, \ldots, T_{q}, T^{\prime}$ such that $\left|T^{\prime}\right|=k$ and $\left|T_{1}\right|+$ $\cdots+\left|T_{q}\right|=n-k$. They correspond to pairs $(T, c)$, where $T$ is a planar rooted tree of degree $n$ and $c$ is a cut of $T$ such that

$$
P^{c}(T)=T_{1} T_{2} \cdots T_{q} \quad \text { and } \quad R^{c}(T)=T^{\prime} .
$$

Then $T$ is obtained by attaching $T_{1}, T_{2}, \ldots, T_{q}$ (in order) to the vertices of $T^{\prime}$. Let $n_{i}$ be the number of the $T_{j}$ attached to the $i$ th vertex of $T^{\prime}$ : then

$$
\frac{C_{p}(T)}{C_{p}\left(T_{1}\right) \cdots C_{p}\left(T_{q}\right) C_{p}\left(T^{\prime}\right)}=\prod_{i=1}^{k} \frac{\left(\begin{array}{c}
p \\
c_{i}+n_{i}
\end{array}\right)}{\left(\begin{array}{c}
p \\
c_{i}
\end{array}\right)}=\prod_{i=1}^{k} \frac{\left(\begin{array}{c}
p-c_{i} \\
n_{i}
\end{array}\right)}{\left(\begin{array}{c}
c_{i}+n_{i} \\
c_{i}
\end{array}\right)}
$$

where $c_{i}$ is the number of children of the $i$ th vertex of $T^{\prime}$.

Now if we consider all the ways of attaching $T_{1}, \ldots, T_{q}$ to the vertices of $T^{\prime}$ so that $n_{i}$ of them are attached to the $i$ th vertex of $T^{\prime}$, there are

$$
\prod_{i=1}^{k}\left(\begin{array}{c}
c_{i}+n_{i} \\
c_{i}
\end{array}\right)
$$

different configurations: they will generally be distinct as planar rooted trees, but the ratio (6.14) comes out the same. Thus, the sum of (6.14) over all the ways of doing the attachments is

$$
\sum_{n_{1}+\cdots+n_{q}=q} \prod_{i=1}^{k}\left(\begin{array}{c}
p-c_{i} \\
n_{i}
\end{array}\right)
$$

which by the generalized Vandermonde convolution (see [12, p. 248]) equals

$$
\left(\begin{array}{c}
k p-c_{1}-\cdots-c_{k} \\
q
\end{array}\right)=\left(\begin{array}{c}
k p-(k-1) \\
q
\end{array}\right)
$$

since the tree $T^{\prime}$ has a total of $k-1$ edges. But this means that the sum of contributions from terms of $\Delta\left(X_{n}\right)$ to the coefficient of (6.13) is

$$
\left(\begin{array}{c}
k(p-1)+1 \\
q
\end{array}\right) \text {. }
$$




\section{References}

[1] C. Bergbauer and D. Kreimer, Hopf algebras in renormalization theory: locality and Dyson-Schwinger equations from Hochschild cohomology, in Physics and Number Theory (ed. by V. Turaev and L. Nyssens), IRMA Lect. Math. Theor. Phys. 10, European Math. Soc. Publishing House, Zürich, 2006, 133-164.

[2] C. Brouder, Runge-Kutta methods and renormalization, Eur. Phys. J. C 12 (2000), 521-534.

[3] C. Brouder and A. Frabetti, Noncommutative renormalization for massless QED, preprint hep-th/0011161.

[4] C. Brouder and A. Frabetti, QED Hopf algebras on planar binary trees, J. Algebra 267 (2003), 298-322.

[5] R. Ehrenborg, On posets and Hopf algebras, Adv. Math. 119 (1996), $1-25$.

[6] A. Connes and D. Kreimer, Hopf algebras, renormalization, and noncommutative geometry, Comm. Math. Phys. 199 (1998), 203-242.

[7] L. Foissy, Les algèbres de Hopf des arbres enracinés décorés, I, Bull. Sci. Math. 126 (2002), 193-239.

[8] L. Foissy, Les algèbres de Hopf des arbres enracinés décorés, II, Bull. Sci. Math. 126 (2002), 249-288.

[9] L. Geissinger, Hopf algebras of symmetric functions and class functions, in Combinatoire et représentation de groupe symmétrique (Strasbourg, 1976), Lect. Notes in Math. 579, Springer, Berlin, 1977, 168-181.

[10] I. M. Gelfand, D. Krob, A. Lascoux, B. Leclerc, V. S. Retakh, and J.-Y. Thibon, Noncommutative symmetric functions, Adv. Math. 112 (1995), $218-348$.

[11] I. M. Gessel, Multipartite P-partitions and inner products of skew Schur functions, in Combinatorics and Algebra, Contemp. Math. 34, American Math. Soc., Providence, 1984, pp. 289-301. 
[12] R. L. Graham, D. E. Knuth, and O. Patashnik, Concrete Mathematics, 2nd ed., Addison-Wesley, New York, 1994.

[13] R. Grossman and R. G. Larson, Hopf-algebraic structure of families of trees, J. Algebra 126 (1989), 184-210.

[14] M. E. Hoffman, Combinatorics of rooted trees and Hopf algebras, Trans. Amer. Math. Soc. 335 (2003), 3795-3811.

[15] R. Holtkamp, Comparison of Hopf algebras on trees, Arch. Math. (Basel) 80 (2003), 368-383.

[16] J-L. Loday and M. O. Ronco, Hopf algebra of the planar binary trees, Adv. Math. 139 (1998), 293-309.

[17] I. G. MacDonald, Symmetric Functions and Hall Polynomials, Oxford University Press, New York, 1995.

[18] W. Zhao, A NCS system over the Grossman-Larson Hopf algebra of labeled rooted trees, preprint math.C0/0509136. 